\title{
SOME PROPERTIES OF CLASS $A(k)$ OPERATORS AND THEIR HYPONORMAL TRANSFORMS
}

\author{
J. STELLA IRENE MARY \\ Department of Mathematics, PSG college of Arts and Science, Coimbatore-641014, India \\ e-mail: stellairenemary@yahoo.co.in \\ stellairenemary@gmail.com \\ and S. PANAYAPPAN \\ Department of Mathematics, Goverment Arts college Coimbatore-641018, India \\ e-mail: panayappan@gmail.com
}

(Received 23 June, 2006; revised 18 September, 2006; accepted 27 September, 2006)

\begin{abstract}
In this paper we shall first show that if $\mathrm{T}$ is a class $A(k)$ operator then its operator transform $\hat{T}$ is hyponormal. Secondly we prove some spectral properties of $T$ via $\hat{T}$. Finally we show that $T$ has property $(\beta)$.
\end{abstract}

2000 Mathematics Subject Classification. 47A10, 47A63.

Let $\mathbf{H}$ be a complex Hilbert space and $\mathbf{L}(\mathbf{H})$ the algebra of all bounded linear operators on $\mathbf{H}$. An operator $T \in \mathbf{L}(\mathbf{H})$ has a unique polar decomposition $T=U|T|$ where $|T|=\left(T^{*} T\right)^{\frac{1}{2}}$ and $U$ is the partial isometry satisfying $N(U)=N(T)=N(|T|)$ and $N\left(U^{*}\right)=N\left(T^{*}\right)$.

An operator $T \in \mathbf{L}(\mathbf{H})$ is said to be hyponormal if $T^{*} T \geq T T^{*}$ where $T^{*}$ is the adjoint of $T$. As a generalisation of hyponormal operators, $p$-hyponormal and loghyponormal operators are defined in [2] and [9] respectively. An operator $T$ is said to be $p$-hyponormal if and only if $\left(T^{*} T\right)^{p} \geq\left(T T^{*}\right)^{p}$ for a positive number $p$ and $\log$ hyponormal if and only if $T$ is invertible and $\log \left(T^{*} T\right) \geq \log \left(T T^{*}\right)$. An operator $T$ is said to be of class $A$ if and only if $\left|T^{2}\right| \geq|T|^{2}$. See [9]. As a generalisation of class $A$, class $A(k)$ and class $A(s, t)$ are defined in [9] and [8] respectively. $T$ belongs to class $A(k)$, if and only if $\left(T^{*}|T|^{2 k} T\right)^{\frac{1}{k+1}} \geq|T|^{2}$ where $k>0$. For positive numbers $s$ and $t$, $T$ belongs to class $A(s, t)$ if and only if $\left(\left|T^{*}\right|^{t}|T|^{2 s}\left|T^{*}\right|^{t}\right)^{\frac{t}{s+t}} \geq\left|T^{*}\right|^{2 t}$. In particular a class $A(k, 1)$ operator is a class $A(k)$ operator [18]. It is well known that inequalities $\left(T^{*}|T|^{2 k} T\right)^{\frac{1}{k+1}} \geq|T|^{2}$ and $\left(\left|T^{*}\right||T|^{2 k}\left|T^{*}\right|\right)^{\frac{1}{k+1}} \geq\left|T^{*}\right|^{2}$ are equivalent [18].

The following inclusion relations hold among these classes:

$$
\begin{aligned}
\{\text { hyponormal }\} & \subset\{p \text {-hyponormal, } 0<p<1\}[\mathbf{1 2}] \\
& \subset\{\operatorname{class} A(s, t), s, t \in(0,1]\}[\mathbf{8}] \\
& \subset\{\operatorname{class} A\}[\mathbf{1 2}] \\
& \subset\{\operatorname{class} A(k), k \geq 1\}[\mathbf{9}] .
\end{aligned}
$$

The Aluthge transform $\tilde{T}=|T|^{\frac{1}{2}} U|T|^{\frac{1}{2}}$ was introduced in [1]. An operator is $w$ hyponormal if $|\tilde{T}| \geq|T| \geq\left|\tilde{T}^{*}\right|$ [3]. The Aluthge transforms are useful in the study of these new classes of operators. "The Aluthge transform is an operator transform from the class of $w$-hyponormal and semi-hyponormal operators to the class of 
semi-hyponormal and hyponormal respectively. By using Aluthge transforms we can obtain spectral properties of these new classes of operators from those of hyponormal operators" [7]. But so far we have not obtained any property of a class $A(k)$ operator and it becomes difficult to study its properties. In this paper a new operator transform $\hat{T}$ of $T$ from the class $A(k)$ to the class of hyponormal operators is given by

$$
|\hat{T}|=\left.\left.|| T\right|^{k} T\right|^{\frac{1}{k+1}}
$$

We denote the spectrum, the point spectrum, the approximate point spectrum and the residual spectrum of an operator $T$ by $\sigma(T), \sigma_{p}(T), \sigma_{a}(T)$ and $\sigma_{r}(T)$ respectively. A complex number $\lambda$ is in the normal approximate point spectrum $\sigma_{n a}(T)$ if there exists a sequence $\left\{y_{n}\right\}$ of unit vectors such that $(T-\lambda) y_{n} \rightarrow 0$ and $(T-\lambda)^{*} y_{n} \rightarrow 0$ as $n \rightarrow \infty$. For a hyponormal operator $T, \sigma_{a}(T)=\sigma_{n a}(T)$ because the inequality $\left\|(T-\lambda)^{*} y\right\| \leq\|(T-\lambda) y\|$ always hold for all $\lambda \in \mathbf{C}$ and all $y \in \mathbf{H}$ [7].

In the following theorem we shall show that the operator transform $\hat{T}$ is hyponormal when $T$ is a class $A(k)$ operator, where $k>1$. Throughout this paper we assume that $k>1$.

THEOREM 1. If $T=U|T|$ is the polar decomposition of a class A(k) operator, then $\hat{T}=W U \|\left.\left. T\right|^{k} T\right|^{\frac{1}{k+1}}$ is hyponormal, where $|T|\left|T^{*}\right|=W|| T \| T^{*}||$ is the polar decomposition.

The following theorems play an important role in the proof of Theorem 1 .

Theorem $\mathbf{R}_{\mathbf{1}}$ [12]. Let $A$ and $B$ be positive operators. Then for each $p \geq 0$ and $r \geq 0$ the following assertions hold:

(a) If $\left(B^{\frac{r}{2}} A^{p} B^{\frac{r}{2}}\right)^{\frac{r}{p+r}} \geq B^{r}$, then $A^{p} \geq\left(A^{\frac{p}{2}} B^{r} A^{\frac{p}{2}}\right)^{\frac{p}{p+r}}$.

(b) If $A^{p} \geq\left(A^{\frac{p}{2}} B^{r} A^{\frac{p}{2}}\right)^{\frac{p}{p+r}}$ and $N(A) \subset N(B)$, then $\left(B^{\frac{r}{2}} A^{p} B^{\frac{r}{2}}\right)^{\frac{r}{p+r}} \geq B^{r}$.

Theorem $\mathbf{R}_{2}$ (Löwner-Heinz inequality [12]). $A \geq B \geq 0$ ensures that $A^{\alpha} \geq B^{\alpha}$ for any $\alpha \in(0,1]$.

Theorem R $\mathbf{R}_{3}$ [13]. Let $T=U|T|$ and $S=V|S|$ and $|T|\left|S^{*}\right|=W|| T|| S^{*}||$ be the polar decompositions. Then $T S=U W V|T S|$ is also the polar decomposition.

Proof of Theorem 1. By assumption $T$ is a class $A(k)$ operator. The following inequalities hold.

$$
\left(T^{*}|T|^{2 k} T\right)^{\frac{1}{k+1}}=\left(|T| U^{*}|T|^{2 k} U|T|\right)^{\frac{1}{k+1}} \geq|T|^{2} \Longleftrightarrow\left(\left|T^{*}\right||T|^{2 k}\left|T^{*}\right|\right)^{\frac{1}{k+1}} \geq\left|T^{*}\right|^{2} .
$$

Applying Theorem $R_{1}$ we obtain

$$
|T|^{2 k} \geq\left(|T|^{k}\left|T^{*}\right|^{2}|T|^{k}\right)^{\frac{k}{k+1}}
$$

Since $\frac{1}{k}<1$, by Theorem $R_{2}$ we have

$$
|T|^{2} \geq\left(|T|^{k}\left|T^{*}\right|^{2}|T|^{k}\right)^{\frac{1}{k+1}}=\left(|T|^{k} U|T|^{2} U^{*}|T|^{k}\right)^{\frac{1}{k+1}} .
$$

From (1) and (3) we get

$$
\left(|T| U^{*}|T|^{2 k} U|T|\right)^{\frac{1}{k+1}} \geq|T|^{2} \geq\left(|T|^{k} U|T|^{2} U^{*}|T|^{k}\right)^{\frac{1}{k+1}}
$$

Let $S=|T|^{k} U|T|=|T|^{k} T$. Then (4) becomes, $\left(S^{*} S\right)^{\frac{1}{k+1}} \geq\left(S S^{*}\right)^{\frac{1}{k+1}}$. This shows that $S=|T|^{k} T$ is $\frac{1}{k+1}$ hyponormal. Besides, since $T=U|T|$ and $|T|^{k}=U^{*} U|T|^{k}$ are the 
polar decompositions, by Theorem $R_{3},|T|^{k} T$ has the following polar decomposition

$$
|T|^{k} T=U^{*} U W U \|\left. T\right|^{k} T \mid
$$

where $|T|^{k}\left|T^{*}\right|=\left.W|| T\right|^{k}\left|T^{*}\right| \mid$ is the polar decomposition. Accordingly we have $N(U) \subseteq N\left(\left|T^{*}\right||T|^{k}\right)=N\left(W^{*}\right)$ and $W^{*} U^{*} U=W^{*}$ on $\mathbf{H}=N(U) \oplus R\left(U^{*}\right)$.

Hence (5) can be written as $|T|^{k} U|T|=U^{*} U W U \|\left. T\right|^{k} U|T||=W U||T|^{k} T \mid$ which is $\frac{1}{k+1}$ hyponormal. It follows that $\hat{T}=W U \|\left.\left. T\right|^{k} T\right|^{\frac{1}{k+1}}$ is hyponormal.

We note that $\left.\left.\hat{T}|| T\right|^{k} T\right|^{\frac{k}{k+1}}=\hat{T}|\hat{T}|^{k}=\left.\left.W U|| T\right|^{k} T|=| T\right|^{k} T$.

THEOREM 2. Let $T$ be a class $A(k)$ operator and $\left\{y_{n}\right\}$ be a sequence of unit vectors in $\boldsymbol{H}$ such that $\lim _{n \rightarrow \infty}(\hat{T}-\lambda) y_{n}=0$. If $\lim _{n \rightarrow \infty}|\hat{T}|^{k} y_{n}$ and $\lim _{n \rightarrow \infty}|T|^{k} y_{n}$ exist, then $\lim _{n \rightarrow \infty}(T-\lambda) y_{n}=0$ and $\lim _{n \rightarrow \infty}(T-\lambda)^{*} y_{n}=0$ where $\lambda \in \boldsymbol{C}$.

Proof. Since $\hat{T}$ is hyponormal, $\lim _{n \rightarrow \infty}(\hat{T}-\lambda) y_{n}=0$ implies that $\lim _{n \rightarrow \infty}$ $(\hat{T}-\lambda)^{*} y_{n}=0$. When $\lambda=0, \lim _{n \rightarrow \infty} \hat{T} y_{n}=0$ and hence $\lim _{n \rightarrow \infty}\left\|\hat{T} y_{n}\right\|=0$. Since $T$ is a class $A(k)$ operator we have

$$
\begin{aligned}
\left\|T y_{n}\right\|^{2} & =\left(|T|^{2} y_{n}, y_{n}\right) \\
& \leq\left(\left(T^{*}|T|^{2 k} T\right)^{1 / k+1} y_{n}, y_{n}\right) \\
& =\left(\|\left.\left. T\right|^{k} T\right|^{2 / k+1} y_{n}, y_{n}\right) \\
& =\left\|\hat{T} y_{n}\right\|^{2} \quad \text { since } \hat{T}=W U \|\left.\left. T\right|^{k} T\right|^{1 / k+1} .
\end{aligned}
$$

It follows that $\lim _{n \rightarrow \infty}\left\|T y_{n}\right\| \leq \lim _{n \rightarrow \infty}\left\|\hat{T} y_{n}\right\|=0$ and hence $\lim _{n \rightarrow \infty} T y_{n}=0$.

Also, since $\left\|T^{*} y_{n}\right\| \leq\left\|T y_{n}\right\|$, we have $\lim _{n \rightarrow \infty}\left\|T^{*} y_{n}\right\| \leq \lim _{n \rightarrow \infty}\left\|T y_{n}\right\|=0$ and hence $\lim _{n \rightarrow \infty} T^{*} y_{n}=0$.

On the other hand, when $\lambda \neq 0$ we have $\lim _{n \rightarrow \infty}(\hat{T}-\lambda) y_{n}=0$ and $\lim _{n \rightarrow \infty}$ $(\hat{T}-\lambda)^{*} y_{n}=0$ so that

$$
\left.\lim _{n \rightarrow \infty}\left(|\hat{T}|^{2}-|\lambda|^{2}\right) y_{n}=0 \text { and } \lim _{n \rightarrow \infty}\left|(\hat{T})^{*}\right|^{2}-|\lambda|^{2}\right) y_{n}=0 .
$$

Since $|\hat{T}|^{2}=\left.\left.|| T\right|^{k} T\right|^{\frac{2}{k+1}}=\left(T^{*}|T|^{2 k} T\right)^{\frac{1}{k+1}} \quad$ and $\quad\left|(\hat{T})^{*}\right|^{2}=\left.\left.\left|T^{*}\right| T\right|^{k}\right|^{\frac{2}{k+1}}=\left(|T|^{k}\left|T^{*}\right|^{2}\right.$ $\left.|T|^{k}\right)^{\frac{1}{k+1}}$, we obtain from (6) that

$$
\lim _{n \rightarrow \infty}\left(\left(T^{*}|T|^{2 k} T\right)^{\frac{1}{k+1}}-|\lambda|^{2}\right) y_{n}=0 \text { and } \lim _{n \rightarrow \infty}\left(\left(|T|^{k}\left|T^{*}\right|^{2}|T|^{k}\right)^{\frac{1}{k+1}}-|\lambda|^{2}\right) y_{n}=0 .
$$

Since $T$ belongs to class $A(k)$,

$$
\left(T^{*}|T|^{2 k} T\right)^{\frac{1}{k+1}} \geq|T|^{2} \geq\left(|T|^{k}\left|T^{*}\right|^{2}|T|^{k}\right)^{\frac{1}{k+1}},
$$

and hence by (7) we have

$$
\lim _{n \rightarrow \infty}\left(\left(|T|^{2}-|\lambda|^{2}\right) y_{n}, y_{n}\right)=0
$$

Also,

$$
\left\|\left[\left(T^{*}|T|^{2 k} T\right)^{\frac{1}{k+1}}-|T|^{2}\right]^{\frac{1}{2}} y_{n}\right\|^{2}=\left(\left[\left(T^{*}|T|^{2 k} T\right)^{\frac{1}{k+1}}-|\lambda|^{2}\right] y_{n}, y_{n}\right)-\left(\left[|T|^{2}-|\lambda|^{2}\right] y_{n}, y_{n}\right) .
$$

It follows from (7) and (8) that $\lim _{n \rightarrow \infty}\left\|\left[\left(T^{*}|T|^{2 k} T\right)^{\frac{1}{k+1}}-|T|^{2}\right]^{\frac{1}{2}} y_{n}\right\|^{2}=0$. 
Consequently we obtain

$$
\begin{aligned}
\lim _{n \rightarrow \infty}\left(|T|^{2}-|\lambda|^{2}\right) y_{n}= & \lim _{n \rightarrow \infty}\left[|T|^{2}-\left(T^{*}|T|^{2 k} T\right)^{1 / k+1}\right] y_{n} \\
& +\lim _{n \rightarrow \infty}\left[\left(T^{*}|T|^{2 k} T\right)^{1 / k+1}-|\lambda|^{2}\right] y_{n}=0 .
\end{aligned}
$$

Hence $\lim _{n \rightarrow \infty}(|T|-|\lambda|) y_{n}=0$. By hypothesis $\lim _{n \rightarrow \infty}|T|^{k} y_{n}$ and $\lim _{n \rightarrow \infty}|\hat{T}|^{k} y_{n}$ exist, so that we get

$$
\begin{gathered}
\lim _{n \rightarrow \infty}\left(|T|^{k}-|\lambda|^{k}\right) y_{n}=0, \\
\lim _{n \rightarrow \infty}\left(|\hat{T}|^{k}-|\lambda|^{k}\right) y_{n}=0 .
\end{gathered}
$$

Now $|T|^{k} T=\left.W U|| T\right|^{k} T \mid$ and $\hat{T}=\left.\left.W U|| T\right|^{k} T\right|^{1 / k+1}=W U|\hat{T}|$ implies $|T|^{k} T=$ $\hat{T}|\hat{T}|^{k}$. Hence $T^{*}|T|^{k}=|\hat{T}|^{k}(\hat{T})^{*}$ and so by (9) and (10)

$$
\left(T^{*}-\bar{\lambda}\right) y_{n}=\frac{T^{*}}{|\lambda|^{k}}\left(|\lambda|^{k}-|T|^{k}\right) y_{n}+\frac{|\hat{T}|^{k}}{|\lambda|^{k}}\left((\hat{T})^{*}-\bar{\lambda}\right) y_{n}+\frac{\bar{\lambda}}{|\lambda|^{k}}\left(|\hat{T}|^{k}-|\lambda|^{k}\right) y_{n} \longrightarrow 0
$$

as $n \rightarrow \infty$. That is $\lim _{n \rightarrow \infty}(T-\lambda)^{*} y_{n}=0$. Since $\left|\left\|T^{*} y_{n}\right\|-\right| \lambda|| \leq\left\|(T-\lambda)^{*} y_{n}\right\|$, we have

$$
\lim _{n \rightarrow \infty}\left\|T^{*} y_{n}\right\|=|\lambda|
$$

Also

$$
\begin{aligned}
\left\|\left(T T^{*}-|\lambda|^{2}\right)^{1 / 2} y_{n}\right\|^{2} & =\left(\left(T T^{*}-|\lambda|^{2}\right) y_{n}, y_{n}\right) \\
& =\left(T T^{*} y_{n}, y_{n}\right)-|\lambda|^{2} \\
& =\left\|T^{*} y_{n}\right\|^{2}-|\lambda|^{2}
\end{aligned}
$$

and by (11)

$$
\lim _{n \rightarrow \infty}\left(\left(T T^{*}-|\lambda|^{2}\right) y_{n}, y_{n}\right)=0
$$

Hence by (12) and (8),

$$
\begin{aligned}
\lim _{n \rightarrow \infty}\left\|\left(\left|T^{*}\right|^{2}-|T|^{2}\right)^{1 / 2} y_{n}\right\|^{2} & =\lim _{n \rightarrow \infty}\left(\left(\left|T^{*}\right|^{2}-|T|^{2}\right) y_{n}, y_{n}\right) \\
& =\lim _{n \rightarrow \infty}\left[\left(\left(\left|T^{*}\right|^{2}-|\lambda|^{2}\right) y_{n}, y_{n}\right)-\left(\left(|T|^{2}-|\lambda|^{2}\right) y_{n}, y_{n}\right)\right] \\
& =0 .
\end{aligned}
$$

It follows that

$$
\lim _{n \rightarrow \infty}\left(\left|T^{*}\right|^{2}-|T|^{2}\right) y_{n}=0
$$

By (13) $\quad \lim _{n \rightarrow \infty}\left(\left|T^{*}\right|^{2}-|\lambda|^{2}\right) y_{n}=\lim _{n \rightarrow \infty}\left[\left(\left|T^{*}\right|^{2}-|T|^{2}\right) y_{n}+\left(|T|^{2}-|\lambda|^{2}\right)\right] y_{n}=0$. Finally, $\lim _{n \rightarrow \infty}(T-\lambda) y_{n}=\lim _{n \rightarrow \infty}(\bar{\lambda})^{-1}\left[\left(\left|T^{*}\right|^{2}-|\lambda|^{2}\right) y_{n}-T\left(T^{*}-\bar{\lambda}\right) y_{n}\right]=0$.

COROLlaRY 3. Let $T$ be a class $A(k)$ operator. Suppose that $\lambda \in \sigma_{n a}(\hat{T})$ and $\left\{y_{n}\right\}$ is a corresponding sequence of unit vectors such that $(\hat{T}-\lambda) y_{n} \rightarrow 0$ and $(\hat{T}-\lambda)^{*} y_{n} \rightarrow 0$ as $n \rightarrow \infty$. If $\lim _{n \rightarrow \infty}|\hat{T}|^{k} y_{n}$ and $\lim _{n \rightarrow \infty}|T|^{k} y_{n}$ exist, then $\sigma_{n a}(\hat{T}) \subseteq \sigma_{n a}(T)$. 
Proof. By hypothesis, $\lambda \in \sigma_{n a}(\hat{T}) \Longrightarrow \lim _{n \rightarrow \infty}(\hat{T}-\lambda) y_{n}=0$ and by Theorem 2 $\lim _{n \rightarrow \infty}(T-\lambda) y_{n}=0$ and $\lim _{n \rightarrow \infty}(T-\lambda)^{*} y_{n}=0$.

That is $\lambda \in \sigma_{n a}(T)$. Hence $\sigma_{n a}(\hat{T}) \subseteq \sigma_{\text {na }}(T)$.

THEOREM 4. Let $T$ be a class $A(k)$ operator and $\left\{y_{n}\right\}$ be a sequence of unit vectors in $\boldsymbol{H}$ such that $\lim _{n \rightarrow \infty}|\hat{T}|^{k} y_{n}$ and $\lim _{n \rightarrow \infty}|T|^{k} y_{n}$ exist then $\lim _{n \rightarrow \infty}(T-\lambda) y_{n}=0$ and $\lim _{n \rightarrow \infty}(T-\lambda)^{*} y_{n}=0 \Longrightarrow \lim _{n \rightarrow \infty}(\hat{T}-\lambda) y_{n}=0$, where $\lambda \in \boldsymbol{C}$.

Proof. When $\lambda=0$ we have

$$
\begin{aligned}
\left\|\hat{T} y_{n}\right\|^{2} & =\left.\left.\|W U\| T\right|^{k} T\right|^{\frac{1}{k+1}} y_{n} \|^{2} \\
& =\left(\|\left.\left. T\right|^{k} T\right|^{\frac{2}{k+1}} y_{n}, y_{n}\right) \\
& \leq\left(T^{*}|T|^{2 k} T y_{n}, y_{n}\right)^{\frac{1}{k+1}} .
\end{aligned}
$$

Since $\lim _{n \rightarrow \infty} T y_{n}=0$ we have $\lim _{n \rightarrow \infty}\left(T^{*}|T|^{2 k} T y_{n}, y_{n}\right)=0$. Also from (14) we have $\lim _{n \rightarrow \infty}\left\|\hat{T} y_{n}\right\|=0$ and $\lim _{n \rightarrow \infty} \hat{T} y_{n}=0$. When $\lambda \neq 0$, by hypothesis $\lim _{n \rightarrow \infty}(T-\lambda) y_{n}=0$ and $\lim _{n \rightarrow \infty}(T-\lambda)^{*} y_{n}=0$. It follows that

$$
\lim _{n \rightarrow \infty}\left(|T|^{2}-|\lambda|^{2}\right) y_{n}=0 \text { and } \lim _{n \rightarrow \infty}(|T|-|\lambda|) y_{n}=0 .
$$

By the continuity of operators we have the following equations

$$
\begin{aligned}
& \lim _{n \rightarrow \infty}\left(|T|^{k}-|\lambda|^{k}\right) y_{n}=0, \\
& \lim _{n \rightarrow \infty}\left(|T|^{k} T-|\lambda|^{k} \lambda\right) y_{n}=0, \\
& \lim _{n \rightarrow \infty}\left(T^{*}|T|^{k}-|\lambda|^{k} \bar{\lambda}\right) y_{n}=0, \\
& \lim _{n \rightarrow \infty}\left(\left(T^{*}|T|^{k}|T|^{k} T\right)-|\lambda|^{2(k+1)}\right) y_{n}=0, \\
& \lim _{n \rightarrow \infty}\left(\left.|T|^{k} T\right|^{2}-|\lambda|^{2(k+1)}\right) y_{n}=0, \\
& \lim _{n \rightarrow \infty}\left(\left.\left.|| T\right|^{k} T\right|^{\frac{2}{k+1}}-|\lambda|^{2}\right) y_{n}=0 .
\end{aligned}
$$

That is $\lim _{n \rightarrow \infty}\left(|\hat{T}|^{2}-|\lambda|^{2}\right) y_{n}=0$ and $\lim _{n \rightarrow \infty}(|\hat{T}|-|\lambda|) y_{n}=0$. By hypothesis, $\lim _{n \rightarrow \infty}|\hat{T}|^{k} y_{n}$ exists and hence,

$$
\lim _{n \rightarrow \infty}\left(|\hat{T}|^{k}-|\lambda|^{k}\right) y_{n}=0
$$

Since $\hat{T}|\hat{T}|^{k}=|T|^{k} T$ we have $(\hat{T}-\lambda) y_{n}=(-) \frac{\hat{T}}{|\lambda|^{k}}\left(|\hat{T}|^{k}-|\lambda|^{k}\right) y_{n}+\frac{|T|^{k}}{|\lambda|^{k}}(T-\lambda) y_{n}+$ $\frac{\lambda}{|\lambda|^{k}}\left(|T|^{k}-|\lambda|^{k}\right) y_{n}$. Consequently by (15) and (16) we get $\lim _{n \rightarrow \infty}(\hat{T}-\lambda) y_{n}=0$.

Corollary 5. Let $T$ be a class $A(k)$ operator. Suppose $\lambda \in \sigma_{n a}(T)$ and $\left\{y_{n}\right\}$ is a corresponding sequence of unit vectors such that $(T-\lambda) y_{n} \rightarrow 0$ and $(T-\lambda)^{*} y_{n} \rightarrow 0$ as $n \rightarrow \infty$. If $\lim _{n \rightarrow \infty}|\hat{T}|^{k} y_{n}$ and $\lim _{n \rightarrow \infty}|T|^{k} y_{n}$ exist, then $\sigma_{n a}(T) \subseteq \sigma_{n a}(\hat{T})$.

Proof. By hypothesis, $\lambda \in \sigma_{n a}(T) \Longrightarrow \lim _{n \rightarrow \infty}(T-\lambda) y_{n}=0$ and $\lim _{n \rightarrow \infty}(T-$ $\lambda)^{*} y_{n}=0$. By Theorem $4 \lim _{n \rightarrow \infty}(\hat{T}-\lambda) y_{n}=0$. That is $\lambda \in \sigma_{n a}(\hat{T})$. Hence $\sigma_{n a}(T) \subseteq$ $\sigma_{n a}(\hat{T})$

In the following theorem we shall show that for a class $A(k)$ operator $T, \sigma_{a}(T)=$ $\sigma_{n a}(T)$. 
THEOREM 6. Let $T$ be a class A(k) operator. Suppose $\left\{y_{n}\right\}$ is a sequence of unit vectors in $\boldsymbol{H}$ such that $(T-\lambda) y_{n} \rightarrow 0$ and $\left\||\hat{T}|^{2} y_{n}\right\|-|\lambda|^{2} \rightarrow 0$ as $n \rightarrow \infty$, then $\lim _{n \rightarrow \infty}(T-$ $\lambda)^{*} y_{n}=0$.

Proof. By assumption $\lim _{n \rightarrow \infty}(T-\lambda) y_{n}=0$. Since $\left|\left\|T y_{n}\right\|-\right| \lambda|| \leq\left\|(T-\lambda) y_{n}\right\|$ we obtain $\lim _{n \rightarrow \infty}\left\|T y_{n}\right\|=|\lambda|$. Also $T$ is a class $A(k)$ operator implies that

$$
\begin{aligned}
\left\|T y_{n}\right\|^{2} & =\left(|T|^{2} y_{n}, y_{n}\right) \\
& \leq\left(\left(T^{*}|T|^{2 k} T\right)^{\frac{1}{k+1}} y_{n}, y_{n}\right) \\
& =\left(\left.\left.|| T\right|^{k} T\right|^{\frac{2}{k+1}} y_{n}, y_{n}\right) \\
& \leq\left\|\left.\left.|| T\right|^{k} T\right|^{\frac{2}{k+1}} y_{n}\right\| \text { (Cauchy-Schwarz inequality) } \\
& =\left\||\hat{T}|^{2} y_{n}\right\| .
\end{aligned}
$$

That is $\lim _{n \rightarrow \infty}\left\|T y_{n}\right\|^{2} \leq \lim _{n \rightarrow \infty}\left\||\hat{T}|^{2} y_{n}\right\|$ and so $|\lambda|^{2} \leq \lim _{n \rightarrow \infty}\left\||\hat{T}|^{2} y_{n}\right\|$.

By hypothesis $\lim _{n \rightarrow \infty}\left\||\hat{T}|^{2} y_{n}\right\|=|\lambda|^{2}$ and hence we obtain

$$
\lim _{n \rightarrow \infty}\left(\left.\left.|| T\right|^{k} T\right|^{\frac{2}{k+1}} y_{n}, y_{n}\right)=|\lambda|^{2} .
$$

Now by (17)

$$
\lim _{n \rightarrow \infty}\left\|\left(\|\left.\left. T\right|^{k} T\right|^{\frac{2}{k+1}}-|\lambda|^{2}\right)^{\frac{1}{2}} y_{n}\right\|^{2}=\lim _{n \rightarrow \infty}\left(\|\left.\left. T\right|^{k} T\right|^{\frac{2}{k+1}} y_{n}, y_{n}\right)-|\lambda|^{2}=0 .
$$

It follows that

$$
\lim _{n \rightarrow \infty}\left(\left.\left.|| T\right|^{k} T\right|^{\frac{2}{k+1}}-|\lambda|^{2}\right) y_{n}=0 .
$$

Also

$$
\lim _{n \rightarrow \infty}\left\|\left(\left.\left.|| T\right|^{k} T\right|^{\frac{2}{k+1}}-|T|^{2}\right)^{\frac{1}{2}} y_{n}\right\|^{2}=\lim _{n \rightarrow \infty}\left[\left(\left.\left.|| T\right|^{k} T\right|^{\frac{2}{k+1}} y_{n}, y_{n}\right)-\left(|T|^{2} y_{n}, y_{n}\right)\right]=0,
$$

and hence we obtain

$$
\lim _{n \rightarrow \infty}\left(\left.\left.|| T\right|^{k} T\right|^{\frac{2}{k+1}}-|T|^{2}\right) y_{n}=0
$$

From (18) and (19) we get

$$
\lim _{n \rightarrow \infty}\left(|T|^{2}-|\lambda|^{2}\right) y_{n}=\lim _{n \rightarrow \infty}\left(|T|^{2}-\left.\left.|| T\right|^{k} T\right|^{2 / k+1}\right) y_{n}+\lim _{n \rightarrow \infty}\left(\left.\left.|| T\right|^{k} T\right|^{\frac{2}{k+1}}-|\lambda|^{2}\right) y_{n}=0 .
$$

As a consequence,

$$
\lim _{n \rightarrow \infty}(T-\lambda)^{*} y_{n}=\frac{1}{\lambda} \lim _{n \rightarrow \infty}\left[\left(|T|^{2}-|\lambda|^{2}\right) y_{n}-T^{*}(T-\lambda) y_{n}\right]=0 .
$$

Hence $\lambda \in \sigma_{\text {na }}(T)$.

TheOREM 7. Let $T$ be a class $A(k)$ operator. Suppose $\lambda \in \sigma_{a}(T)$ and $\left\{y_{n}\right\}$ is a corresponding sequence of unit vectors sucht that $(T-\lambda) y_{n} \rightarrow 0$ and $\left\||\hat{T}|^{2} y_{n}\right\|-|\lambda|^{2} \rightarrow$ 0 as $n \rightarrow \infty$ then $\sigma(T)=\sigma(\hat{T})$

To prove Theorem 7 we need the following theorems. 


\section{Theorem $R_{4}$ [11]}

1. If $A$ is normal, then for any $B \in L(H), \sigma(A B)=\sigma(B A)$.

2. Let $T=U|T|$ be the polar decomposition of a p-hyponormal operator $(p>0)$. Then for any $t>0, \sigma\left(U|T|^{t}\right)=\left\{e^{i \theta} \rho^{t}: e^{i \theta} \rho \in \sigma(T)\right\}$.

Theorem $R_{5}$ [17] Let $R$ be a subset of the complex plane $C, T(t)$ an operator-valued function of $t \in[0,1]$ that is continuous in the norm topology, $\tau_{t}, t \in[0,1]$, a family of bijective mappings from $R$ onto $\tau_{t}(R) \subset C$ and, for any fixed $z \in R, \tau_{t}(z)$ is a continuous function of $t \in[0,1]$ such that $\tau_{0}$ is the identity function. Suppose that

$$
\sigma_{a}(T(t)) \cap \tau_{t}(R)=\tau_{t}\left(\sigma_{a}(T(0)) \cap R\right)
$$

for all $t \in[0,1]$. Then for all $t \in[0,1]$,

$$
\begin{gathered}
\sigma_{r}(T(t)) \cap \tau_{t}(R)=\tau_{t}\left(\sigma_{r}(T(0)) \cap R\right), \\
\sigma(T(t)) \cap \tau_{t}(R)=\tau_{t}(\sigma(T(0)) \cap R) .
\end{gathered}
$$

Let $F$ be the set of all strictly monotone increasing continuous nonnegative functions on $R^{+}=[0, \infty)$. Let $F_{0}=\{\Psi \in F: \Psi(0)=0\}$ and $T=U|T|$. For $\Psi \in F_{0}$, the mapping $\widetilde{\Psi}$ is defined by $\widetilde{\Psi}\left(\rho e^{i \theta}\right)=e^{i \theta} \Psi(\rho)$ and $\widetilde{\Psi}(T)=U \Psi(|T|)$.

Theorem $R_{6}[6] \quad$ Let $T=U|T|$ and $\Psi \in F_{0}$. Then $\sigma_{n a}(\widetilde{\Psi}(T))=\widetilde{\Psi}\left(\sigma_{n a}(T)\right)$.

Proof. Let $T=U|T|$ be the polar decomposition of $T$. We shall prove that if $T$ is class $A(k)$, then $\sigma\left(U|T|^{k+1}\right)=\left\{\rho^{k+1} e^{i \theta}: \rho e^{i \theta} \in \sigma(T)\right\}$. Let $T(t)=U|T|^{k+t}$ and $\tau_{t}\left(\rho e^{i \theta}\right)=e^{i \theta} \rho^{k+t}$. Since $|T(t)|=|T|^{k+t}$ and $\left|T(t)^{*}\right|=\left|T^{*}\right|^{k+t}$ we have the following implications.

$$
\begin{aligned}
& T \text { belongs to class } A(k), \Leftrightarrow\left(\left|T^{*}\right||T|^{2 k}\left|T^{*}\right|\right)^{\frac{1}{k+1}} \geq\left|T^{*}\right|^{2} \\
& \Leftrightarrow\left(\left|T(t)^{*}\right|^{\frac{1}{k+t}}|T(t)|^{\frac{2 k}{k+t}}\left|T(t)^{*}\right|^{\frac{1}{k+t}}\right)^{\frac{1}{k+1}} \geq\left|T(t)^{*}\right|^{\frac{2}{k+t}} \\
& \Leftrightarrow T(t) \text { belongs to class } A\left(\frac{k}{k+t}, \frac{1}{k+t}\right) \\
& \Rightarrow T(t) \text { belongs to class } A(k) .
\end{aligned}
$$

By Theorem 6 and Theorem $R_{6}$ we have,

$$
\begin{aligned}
\sigma_{a}(T(t) \backslash\{0\}) & =\sigma_{\text {na }}(T(t) \backslash\{0\}) \\
& =\tau_{t}\left(\sigma_{\text {na }}(T) \backslash\{0\}\right) \\
& =\tau_{t}\left(\sigma_{a}(T) \backslash\{0\}\right) \\
& =\tau_{t}\left(\sigma_{a}(T)\right) \backslash\{0\}
\end{aligned}
$$

Moreover, if $0 \in \sigma_{a}(T(t))$ then there exists a sequence $\left\{y_{n}\right\}$ of unit vectors such that $U|T|^{k+t} y_{n} \rightarrow 0$ as $n \rightarrow \infty$. Hence, $\left\||T|^{k} y_{n}\right\|^{2}=\left(U|T|^{k+t} y_{n}, U|T|^{k-t} y_{n}\right) \rightarrow 0$, so that, $\lim _{n \rightarrow \infty}|T|^{k} y_{n}=0$. It follows that $\lim _{n \rightarrow \infty} T y_{n}=0$ and hence $0 \in \sigma_{a}(T)$.

On the other hand, if $0 \in \sigma_{a}(T)$ then we have $0 \in \sigma_{a}(T(t))$ since,

$$
\left\|U|T|^{k+t} y_{n}\right\|=\left\|U|T||T|^{k+t-1} y_{n}\right\| \leq\left\||T|^{k+t-1}\right\|\left\|T y_{n}\right\| \rightarrow 0 \text { as } n \rightarrow \infty .
$$

Hence we obtain, $\sigma_{a}(T(t))=\tau_{t}\left(\sigma_{a}(T)\right)$ for all $t \in[0,1]$ and by Theorem $R_{5}$, we have $\sigma(T(t))=\tau_{t}(\sigma(T))$ for all $t \in[0,1]$. Putting $t=1$, we get

$$
\sigma\left(U|T|^{k+1}\right)=\left\{\rho^{k+1} e^{i \theta}: \rho e^{i \theta} \in \sigma(T)\right\}
$$


By (1) of Theorem $R_{4}$ and (20) we have,

$$
\begin{aligned}
\sigma\left(W U \|\left. T\right|^{k} T \mid\right) & =\sigma\left(|T|^{k} U|T|\right)=\sigma\left(U|T|^{k+1}\right) \\
& =\left\{\rho^{k+1} e^{i \theta}: \rho e^{i \theta} \in \sigma(T)\right\} .
\end{aligned}
$$

By Theorem 1, $\hat{T}=W U \|\left.\left. T\right|^{k} T\right|^{\frac{1}{k+1}}$ is hyponomal. Hence by Theorem $R_{4}$ we get,

$$
\begin{aligned}
\sigma(\hat{T}) & =\sigma\left(W U \|\left.\left. T\right|^{k} T\right|^{\frac{1}{k+1}}\right)=\left\{\left(\rho^{k+1}\right)^{\frac{1}{k+1}} e^{i \theta}: \rho^{k+1} e^{i \theta} \in \sigma\left(U|T|^{k+1}\right)\right\} \\
& =\left\{\rho e^{i \theta}: e^{i \theta} \rho^{k+1} \in \sigma\left(U|T|^{k+1}\right)\right\} \\
& =\sigma(T) .
\end{aligned}
$$

In the following corollaries we assume that $T$ satisfies the following Limit Condition.

Limit Condition. For each $\lambda \in \sigma_{a}(T)$ and a corresponding sequence $\left\{y_{n}\right\}$ of unit vectors, $\hat{T}$ satisfies the condition that $\lim _{n \rightarrow \infty}\left\||\hat{T}|^{2} y_{n}\right\|=|\lambda|^{2}$, where $T$ is a class $A(k)$ operator and $\hat{T}$ is its hyponormal operator transform.

COROLlary 8. Let $T$ be a class $A(k)$ operator such that the Limit Condition is satisfied. Then $\|T\|=\|\hat{T}\|=r(T)$ where $r(T)$ denotes the spectral radius of $T$.

Proof.

$$
\begin{aligned}
\|\hat{T}\| & =\sup \{\|\hat{T} y\|:\|y\|=1\} \\
& =\sup \left\{\left(|\hat{T}|^{2} y, y\right)^{\frac{1}{2}}:\|y\|=1\right\} \\
& =\sup \left\{\left(\|\left.\left. T\right|^{k} T\right|^{\frac{2}{k+1}} y, y\right)^{\frac{1}{2}}:\|y\|=1\right\} \\
& \geq \sup \{\|T y\|:\|y\|=1\} \\
& =\|T\| .
\end{aligned}
$$

Since $\hat{T}$ is hyponormal, $\|\hat{T}\|=r(\hat{T})$. Hence we have,

$$
\begin{aligned}
\|T\| & \leq\|\hat{T}\| \\
& =r(\hat{T}) \\
& =\sup \{|\lambda|: \lambda \in \sigma(\hat{T}\} \\
& =\sup \{|\lambda|: \lambda \in \sigma(T)\} \\
& =r(T) .
\end{aligned}
$$

Since every class $A(k)$ operator is normaloid, $\|T\|=r(T)$. So $\|T\|=r(T)=r(\hat{T})=$ $\|\hat{T}\|$

COROLlARY 9. Let $T$ be a class A(k) operator with a single limit point in its spectrum such that the Limit Condition is satisfied, then the residual spectrum of $T$ is empty.

Proof. By Theorem $7 \sigma(T)=\sigma(\hat{T})$. Hence $\sigma(\hat{T})$ has a single limit point. Since $\hat{T}$ is hyponormal with a single limit point in its spectrum it is normal [16]. For a hyponormal operator the residual spectrum is empty. Since $\sigma_{p}(T)=\sigma_{p}(\hat{T})$ the residual spectrum of $T$ is also empty. 
Corollary 10. A generalised nilpotent class A(k) operator satisfying the Limit Condition is necessarily zero.

Proof. Since $\hat{T}$ is hyponormal, $\sigma(\hat{T})$ contains a scalar $\mu$ such that $|\mu|=\|\hat{T}\|[4]$. For every positive integer $n$, it follows that [10, Theorem 33.1],

$$
\|T\|^{n}=\|\hat{T}\|^{n}=\|\mu\|^{n}=\left\|\mu^{n}\right\| \leq\left\|T^{n}\right\| \leq\|T\|^{n} .
$$

Hence $\|T\|^{n}=\left\|T^{n}\right\|$. By hypothesis, $\lim _{n \rightarrow \infty}\left\|T^{n}\right\|^{\frac{1}{n}}=0$. It follows that $\|T\|=0$. Hence $T=0$.

An operator $T \in \mathbf{L}(\mathbf{H})$ is said to satisfy Single-Valued Extension Property (SVEP) if for any open subset $V$ in $\mathbf{C}$, the function $T-\lambda: \Theta(V, H) \rightarrow \Theta(V, H)$ defined by pointwise multiplication, is one-to-one. Here $\Theta(V, H)$ denotes the Fréchet space of $\mathbf{H}$-valued analytical functions on $V$ with respect to the uniform topology. An operator $T \in L(H)$ is said to satisfy the property $(\beta)$ if for every open subset $\mathbf{G}$ of $\mathbf{C}$ and every sequence $f_{n}: \mathbf{G} \rightarrow \mathbf{H}$ of $\mathbf{H}$-valued analytic functions such that $(T-\lambda) f_{n}(\lambda)$ converges uniformly to 0 in norm on compact subsets of $\mathbf{G}, f_{n}(\lambda)$ converges uniformly to 0 in norm on compact subsets of $\mathbf{G}$. This was first introduced by Bishop [5].

To prove that a class $A(k)$ operator $T$ has property $(\beta)$ we need the following Theorem which is a modified form of [14, Lemma 2.5].

Theorem $\mathbf{R}_{7}[\mathbf{1 4}]$. Let $\boldsymbol{D}$ be an open subset of $\boldsymbol{C}$ and $f_{n}: \boldsymbol{D} \rightarrow \boldsymbol{H}(n=1,2, \ldots)$ vector valued analytic functions such that $|\mu| f_{n}(\mu) \rightarrow 0$ uniformly on every compact subset of $\boldsymbol{D}$. Then $f_{n}(\mu) \rightarrow 0$ again uniformly on every compact subset of $\boldsymbol{D}$.

Proof of Theorem $R_{7}$.

Let us fix an arbitrary $\lambda \in \mathbf{D}$. It suffices to show that there exists a constant $r>0$ such that $\{|\mu-\lambda| \leq r\} \subset \mathbf{D}$ and $f_{n}(\mu) \rightarrow 0$ uniformly on $\{|\mu-\lambda| \leq r\}$. If $\lambda \neq 0$, then we need merely to take $r$ such that $0 \notin\{|\mu-\lambda| \leq r\} \subset \mathbf{D}$. We consider the case in which $\lambda=0$. Take any constant $r>0$ such that $\{|\mu| \leq r\} \subset \mathbf{D}$. Then for each $n=1,2, \ldots$, we can find an $\omega_{n}$ with $\left|\omega_{n}\right|=r$ such that $\left\|f_{n}(\mu)\right\| \leq\left\|f_{n}\left(\omega_{n}\right)\right\|$ on $\{|\mu| \leq r\}$ by the maximum principle. Thus

$$
\left\|f_{n}(\mu)\right\|=\frac{1}{\left|\omega_{n}\right|}\left|\omega_{n}\right|\left\|f_{n}(\mu)\right\| \leq \frac{1}{r}\left\|\omega_{n} f_{n}\left(\omega_{n}\right)\right\| \rightarrow 0
$$

uniformly on $\{|\mu| \leq r\}$.

THEOREM 11. A class $A(k)$ operator $T$ has property $(\beta)$ if $\lim _{n \rightarrow \infty}|T|^{k} f_{n}(\mu)$ and $\lim _{n \rightarrow \infty}|\hat{T}|^{k} f_{n}(\mu)$ both exist and $\lim _{n \rightarrow \infty}\left[\left\||\hat{T}|^{2} f_{n}(\mu)\right\|-\left\||\mu|^{2} f_{n}(\mu)\right\|\right]=0$.

Proof. Let $\mathbf{D}$ be an open neighborhood of $\lambda \in \mathbf{C}$ and $f_{n}(n=1,2, \ldots)$ vector-valued analytic functions on $\mathbf{D}$ such that $(T-\mu) f_{n}(\mu) \rightarrow 0$ uniformly on every compact subset of D.

We may assume that $\sup _{n}\left\|f_{n}(\mu)\right\|<+\infty$ on every compact subset of $\mathbf{D}$. In fact, let $M_{n}$ be a positive number such that $\left\|f_{n}(\mu)\right\| \leq M_{n}$. Then by replacing $f_{n}(\mu)$ with $\frac{f_{n}(\mu)}{M_{n}+1}$, we have $\sup _{n}\left\|f_{n}(\mu)\right\| \leq 1$ and $(T-\mu) f_{n}(\mu) \rightarrow 0$ uniformly on every compact subset of D. By hypothesis, $(T-\mu) f_{n}(\mu) \rightarrow 0$ uniformly on every compact subset of D. Since $\left|\left\|T f_{n}(\mu)\right\|-\left\|\mu f_{n}(\mu)\right\|\right| \leq\left\|(T-\mu) f_{n}(\mu)\right\|$ we obtain

$$
\lim _{n \rightarrow \infty}\left(\left\|T f_{n}(\mu)\right\|-\left\|\mu f_{n}(\mu)\right\|\right)=0 .
$$


Since $T$ belongs to class $A(k)$,

$$
\begin{aligned}
\left\|T f_{n}(\mu)\right\|^{2}-\left\|\mu f_{n}(\mu)\right\|^{2} & =\left(|T|^{2} f_{n}(\mu), f_{n}(\mu)\right)-\left(|\mu|^{2} f_{n}(\mu), f_{n}(\mu)\right) \\
& \leq\left(\left(T^{*}|T|^{2 k} T\right)^{\frac{1}{k+1}} f_{n}(\mu), f_{n}(\mu)\right)-\left(|\mu|^{2} f_{n}(\mu), f_{n}(\mu)\right) \\
& =\left(\left.\left.|| T\right|^{k} T\right|^{\frac{2}{k+1}} f_{n}(\mu), f_{n}(\mu)\right)-\left(|\mu|^{2} f_{n}(\mu), f_{n}(\mu)\right) \\
& =\left(|\hat{T}|^{2} f_{n}(\mu), f_{n}(\mu)\right)-\left(|\mu|^{2} f_{n}(\mu), f_{n}(\mu)\right) \\
& \leq\left[\left\||\hat{T}|^{2} f_{n}(\mu)\right\|-\left\||\mu|^{2} f_{n}(\mu)\right\|\right]\left\|f_{n}(\mu)\right\| \rightarrow 0
\end{aligned}
$$

by assumption. Hence

$$
\lim _{n \rightarrow \infty}\left[\left(|\hat{T}|^{2} f_{n}(\mu), f_{n}(\mu)\right)-\left(|\mu|^{2} f_{n}(\mu), f_{n}(\mu)\right)\right]=0 .
$$

Also

$$
\begin{aligned}
\left\|\left(|\hat{T}|^{2}-|\mu|^{2}\right) f_{n}(\mu)\right\|^{2}= & \left\||\hat{T}|^{2} f_{n}(\mu)\right\|-2|\mu|^{2}\left(|\hat{T}|^{2} f_{n}(\mu), f_{n}(\mu)\right)+|\mu|^{4}\left\|f_{n}(\mu)\right\|^{2} \\
= & \left\||\hat{T}|^{2} f_{n}(\mu)\right\|-\left\||\mu|^{2} f_{n}(\mu)\right\|^{2} \\
& -2|\mu|^{2}\left(\left(|\hat{T}|^{2}-|\mu|^{2}\right) f_{n}(\mu), f_{n}(\mu)\right) \rightarrow 0,
\end{aligned}
$$

uniformly as $n \rightarrow \infty$. That is,

$$
\begin{gathered}
\lim _{n \rightarrow \infty}\left(|\hat{T}|^{2}-|\mu|^{2}\right) f_{n}(\mu)=0, \\
\lim _{n \rightarrow \infty}(|\hat{T}|-|\mu|) f_{n}(\mu)=0 .
\end{gathered}
$$

By hypothesis $\lim _{n \rightarrow \infty}|\hat{T}|^{k} f_{n}(\mu)$ exists and hence

$$
\lim _{n \rightarrow \infty}\left(|\hat{T}|^{k}-|\mu|^{k}\right) f_{n}(\mu)=0 .
$$

By (21) and (22)

$$
\left\|\left(|\hat{T}|^{2}-|T|^{2}\right)^{\frac{1}{2}} f_{n}(\mu)\right\|^{2}=\left(|\hat{T}|^{2} f_{n}(\mu), f_{n}(\mu)\right)-\left(|T|^{2} f_{n}(\mu), f_{n}(\mu)\right) \rightarrow 0 .
$$

Hence $\left(|\hat{T}|^{2}-|T|^{2}\right) f_{n}(\mu) \rightarrow 0$ uniformly. By (23) $\lim _{n \rightarrow \infty}\left(|T|^{2}-|\mu|^{2}\right) f_{n}(\mu)=0$; $\lim _{n \rightarrow \infty}(|T|-|\mu|) f_{n}(\mu)=0$. Hence $\lim _{n \rightarrow \infty}\left(|T|^{k}-|\mu|^{k}\right) f_{n}(\mu)=0$.

Since $\hat{T}|\hat{T}|^{k}=|T|^{k} T$, we have

$$
\begin{aligned}
(\hat{T}-\mu)|\hat{T}|^{k} f_{n}(\mu)= & \left(|T|^{k} T f_{n}(\mu)-\mu|\hat{T}|^{k} f_{n}(\mu)\right) \\
= & |T|^{k}(T-\mu) f_{n}(\mu)+\mu\left(|T|^{k}-|\mu|^{k}\right) f_{n}(\mu) \\
& +\mu\left(|\mu|^{k}-|\hat{T}|^{k}\right) f_{n}(\mu) \rightarrow 0,
\end{aligned}
$$

uniformly. According to Putinar [15], every hyponormal operator has property $(\beta)$ and hence $\hat{T}$ has property $(\beta)$. Hence, $|\hat{T}|^{k} f_{n}(\mu) \rightarrow 0$ uniformly and $|\hat{T}| f_{n}(\mu) \rightarrow 0$ uniformly as $n \rightarrow \infty$. By (24) we have $|\mu| f_{n}(\mu) \rightarrow 0$ uniformly and by Theorem $R_{7}$ we obtain $f_{n}(\mu) \rightarrow 0$ uniformly. Thus $T$ has property $(\beta)$ and hence the Single Valued Extension Property. 


\section{REFERENCES} $307-315$.

1. A. Aluthge, On p-hyponormal operators for $0<p<1$, Int. Eq. Op. Th. 13 (1990),

2. A. Aluthge, Some generalised theorems on $p$-hyponormal operators, Int. Eq. Op. Th. 24 (1996), 497-501.

3. A. Aluthge and Wang, w-hyponormal operators, Int. Eq. Op. Th. 36 (2000), 1-10.

4. S. K. Berberian, A note on hyponormal operators, Pacific J. Math. 12 (1962), 1171-1175. 379-397.

5. E. Bishop, A duality theorem for an arbitrary operator, Pacific J. Math. 9 (1959),

6. M. Chō and M. Itoh, Putnam's inequality for $p$-hyponormal operators, Proc. Amer. Math. Soc 123 (1995), 2435-2440.

7. M. Chō and T. Yamazaki, An operator transform from class A to the class of hyponormal operators and its application, Int. Eq. Op. Th. 53 (2005), 497-508.

8. M. Fujii, D. Jung, S. H. Lee, M. Y. Lee and R. Nakamoto, Some class of operators related to paranormal and log-hyponormal operators, Math. Japan. 51 (2000),395-402.

9. T. Furuta, M. Ito and T. Yamazaki, A subclass of paranormal operators including class of log-hyponormal and several related classes, Sci. Math. 1 (1998), 389-403.

10. P. R. Halmos, Introduction to Hilbert space and the theory of spectral multiplicity (Chelsea, New York, 1951).

11. T. Huruya, A note on p-hyponormal operators, Proc. Amer. Math. Soc. 125 (1997), $3617-3624$.

12. M. Ito and T. Yamazaki, Relations between two inequalities $\left(B^{\frac{r}{2}} A^{p} B^{\frac{r}{2}}\right)^{\frac{r}{p+r}} \geq B^{r}$ and $A \geq\left(A^{\frac{p}{2}} B^{r} A^{\frac{p}{2}}\right)^{\frac{p}{p+r}}$ and their applications, Int. Eq. Op. Th. 44 (2002), 442-450.

13. M. Ito and T. Yamazaki and M. Yanagida, The polar decomposition of the product of operators and its applications, Int. Eq. Op. Th. 49 (2004), 461-472.

14. F. Kimura, Analysis of non-normal operators via Aluthge transformation, Int. Eq. Op. Th. 50 (2004), 375-384. $385-395$.

15. M. Putinar, Hyponormal operarators are subscalar, J. Operator Theory. 12 (1984),

16. J. Stampfli, Hyponormal operators, Pacific J. Math. 12 (1962), 1453-1458.

17. D. Xia, Spectral theory of hyponormal operators (Birkhauser Verlag, Basel, 1983).

18. T. Yamazaki, On powers of class $A(k)$ operators including p-hyponormal and log hyponormal operators, Math. Inequal. Appl. 3 (2000), 97-104. 